\title{
Udo Kelle
}

\section{„Kundenorientierung" in der Altenpflege? Potemkinsche Dörfer sozialpolitischen Qualitätsmanagements}

\section{Einleitung}

Der Strukturwandel des Alterns, insbesondere die wachsende Anzahl von allein lebenden Hochaltrigen, lässt die Zahl von gebrechlichen alten Menschen in institutioneller Betreuung steigen. Der zunehmende Finanzbedarf für deren Versorgung wird die Tendenzen zur Ökonomisierung der Altenpflege und zur Taylorisierung des Pflegehandelns, die mit der Einführung der Pflegeversicherung in den 1990er Jahren beispielsweise in der Festlegung von „Orientierungswerten zur Pflegezeitbemessung“ angelegt waren, weiter verstärken. Private Träger von Pflegeeinrichtungen und gemeinnützige Organisationen werden dabei gleichermaßen in die betriebswirtschaftliche Logik der Reduktion von Personal(kosten) um jeden Preis gezwungen. Hieraus resultierende Missstände von Vernachlässigung und „gefährlicher Pflege“ in vielen Einrichtungen rufen bereits jetzt schon durch Medienberichte die Öffentlichkeit wach und schaffen rechtsstaatliche Legitimationsprobleme, indem sie die Existenz einer wachsenden „Anstaltsbevölkerung“, deren Status als Träger bürgerlicher und allgemeiner Menschenrechte prekär ist, in den Fokus öffentlicher Aufmerksamkeit rücken.

Die Politik hat hierauf in vielen europäischen Ländern mit einer Einführung und Ausweitung von staatlichen und halbstaatlichen Kontrollinstitutionen reagiert, wie sie etwa in Deutschland durch das „Pflegeversicherungsgesetz“ selbst und insbesondere das „Pflegequalitätssicherungsgesetz“ oder in Großbritannien durch den „National Standards Act 2000“ implementiert wurden. Ganz im Sinne der Ökonomisierung des Sozialen verdrängt dabei ein betriebswirtschaftlich orientiertes Leitbild von Qualitätsmanagement traditionelle Orientierungen von religiös oder ethisch motivierter Nächstenliebe, von Subsidiarität und Solidarität. Das Eindringen ingenieurs- und betriebswirtschaftlicher Diskurse über "Qualitätsentwicklung“ und „Qualitätsmanagement" in die „Pflegeindustrie“ (wie dieser Sektor in englischsprachigen Publikationen zunehmend genannt wird) erfordert dabei die exakte Messbarkeit konkreter outcomes. Professionelles Pflegehandeln (etwa: das Waschen und Anziehen hilfs- 
bedürftiger Menschen) findet aber aus guten Gründen in einem gegen uneingeschränkte Beobachtbarkeit geschützten setting statt, in welchem „Vertrauensgüter“" produziert werden, deren wahre Qualität nur schwer messbar ist. Weil aber im Rahmen von Vermarktlichung und Ökonomisierung der Altenpflege der hilfsbedürftige ältere Mensch zunehmend als „Kunde“ verstanden wird, bietet es sich hier an, Pflegequalität durch Kundenbefragungen zu erfassen, wie sie auf vielen Konsumgütermärkten durchgeführt werden.

In diesem Beitrag soll untersucht werden, inwieweit mit solchen Konzepten und rhetorischen Figuren ein verzerrtes und geschöntes Bild sozialer Problemlagen gezeichnet wird. Hierzu soll in einem ersten Schritt die Verwendung von Begriffen wie „Kundenorientierung“ und „Kundenzufriedenheit“ im Kontext der stationären Altenpflege diskutiert und nachgefragt werden, welche sinnvolle Funktion die Kennzeichnung von abhängigen, anspruchsberechtigten und hilfsbedürftigen älteren Menschen als „Kunden“ erfüllen kann. Welch problematische Folgen die unkritische Verwendung eines Kundenbegriffs faktisch haben kann, soll dann anhand von empirischen Ergebnissen einer eigenen Studie (Kelle/Niggemann 2002, 2003) gezeigt werden. Mit einem Methodenmix aus qualitativen und quantitativen Verfahren haben wir untersucht, inwieweit gängige Messinstrumente zu „Kundenzufriedenheit“ vertrauenswürdige Ergebnisse liefern. Unsere Daten machen deutlich, dass die Messung und Quantifizierung von Pflegequalität durch eine Befragung von dauerhaft institutionalisierten, hilfsbedürftigen und vulnerablen älteren Menschen nahezu zwangsläufig zu schwerwiegenden Missverständnissen und Fehlern führen muss.

\section{Das Konzept der Kundenorientierung in der Pflegeindustrie}

Im Jahr 2003 befanden sich insgesamt etwas mehr als 600.000 Menschen in stationärer Pflege, das sind 3\% aller über 60-Jährigen (vgl. http://www.destatis.de/ download/d/solei/bericht03deutschl.pdf). Mit wachsendem Lebensalter steigt die Chance (oder das Risiko) für eine - dann fast immer dauerhafte Institutionalisierung dramatisch an: liegt der Anteil der in stationärer Pflege lebenden Menschen bei den 65- bis 70-Jährigen noch bei $0,6 \%$, so steigt sie bei den über 90-Jährigen auf 27,7\%. Dabei ist die Statuspassage zwischen der selbstständigen Haushaltsführung und dem Leben in einer stationären Einrichtung zumeist ein schmerzhafter Prozess. Ältere Menschen halten oftmals trotz erheblicher Hilfebedürftigkeit an ihrer vertrauten Wohnumgebung fest, so dass der Schritt in ein Pflegeheim häufig schlecht vorbereitet, unter mehr oder weniger sanftem Druck der Umgebung und nur bedingt freiwillig erfolgt. Insbesondere eine ungeplante und überstürzte Übersiedlung kann schwerwiegende psychische Probleme, wie Angstzustände, Depressionen oder die bei beginnender Demenz gefürchteten schweren Verwirrtheitszustände, auslösen (vgl. hierzu auch Thiele et al. 2002). 
Zudem gibt die Versorgung in stationären Einrichtungen immer wieder Anlass zu öffentlicher Kritik. Von Zeit zu Zeit erzielen Insiderberichte und Veröffentlichungen investigativer Journalisten, in denen schockierende Aspekte des Pflegealltags beschrieben werden, große Medienresonanz (etwa: Breitscheidel 2005; Fussek/Loerzer 2005). Auch wenn skandalisierende Tendenzen in solchen Berichten von Seiten der Pflegeprofessionen moniert werden (Jenrich/ Schlichting 2006), so wird hierdurch doch verstärkter Handlungsdruck auf staatliche und öffentlich-rechtliche Akteure ausgeübt. Sanktions- und Kontrollinstanzen zur Bearbeitung solcher Probleme, wie der „Medizinische Dienst der Krankenkassen" oder die Heimaufsicht, wurden schon vor geraumer Zeit installiert. Insbesondere nach Einführung der Pflegeversicherung wurden neue juristische und administrative Strukturen und Prozesse geschaffen, um die persönlichen Rechte älterer Menschen zu stärken und die Einhaltung von Qualitätsstandards in der Altenpflege zu sichern. So soll das „Pflegequalitätssicherungsgesetz" (PQsG) Verbraucherrechte der Pflegebedürftigen stärken, indem die Einrichtungen der Altenpflege einerseits auf ein verstärktes einrichtungsinternes Qualitätsmanagement verpflichtet und andererseits die externen Prüfkompetenzen der medizinischen Dienste verstärkt wurden. Die als Durchführungsverordnung zum PQsG geplante „Pflegeprüfverordnung“ ist allerdings 2003 im Bundesrat gescheitert und wurde vom Bundesgesundheitsministerium nicht weiter verfolgt, eine Novellierung des Pflegeversicherungsgesetzes steht zu erwarten. Während über konkrete Details der zukünftigen gesetzlich vorgegebenen Qualitätsstandards und Prüfprozeduren noch Unsicherheit herrscht, versuchen insbesondere große Träger den vermutlich weiter wachsenden Anforderungen entgegen zu kommen, indem sie Prozesse der Evaluation und Zertifizierung von Pflegequalität selbst initiieren.

Ein weiterer Faktor in diesem Geschehen ist die Finanzierungskrise der sozialen Sicherungssysteme. Seit der Einführung der Pflegeversicherung wird Altenpflege zu einem großen Teil durch Sozialabgaben finanziert - somit wirkt sich die fortdauernde Beschäftigungskrise hier auf gleiche Weise aus wie im System der Gesetzlichen Krankenversicherung. Viele Akteure in der Altenpflege orientieren sich angesichts eines steigenden Bedarfs an Leistungen einerseits und verringerter Finanzierungsoptionen andererseits zunehmend an einer streng betriebswirtschaftlichen Logik von Effizienz und Effektivität. Die mit einem wachsenden staatlichen Kontrollaufwand verbundenen Ansprüche, wonach Pflegeanbieter bei allenfalls stagnierender Finanzierung umfangreichere oder verbesserte Leistungen zu erbringen haben, bilden die Hintergrundfolie, vor der seit einigen Jahren Diskurse um Qualitätsmanagement und Qualitätssicherung ihren Einzug in der Altenpflege gehalten haben. Dass diese aus dem kaufmännischen Bereich stammenden Konzepte vordergründig eine Orientierung an Kundenbedürfnissen und Kundenzufriedenheit verlangen, wird dabei oft als Weg zum „empowerment“ von Pflegebedürftigen betrachtet, deren Stel- 
lung auf diese Weise hierdurch vermeintlich gestärkt werden könne: die „Zufriedenheit“ des „Kunden“ Heimbewohner sei als ein wichtiger Indikator für die Qualität der pflegerischen Versorgung zu betrachten. Damit gerät die „Frage nach der Nutzerperspektive“ (Wingenfeld 2003) verstärkt in den Fokus der Aufmerksamkeit.

Die Verschiebung von einem sozialpolitischen „Klientel-“ oder „Wohlfahrtsdiskurs“ hin zu einem ökonomischen „Kundendiskurs“ hat allerdings ihre Tücken. Eine Vermischung von teilweise unvereinbaren Systemlogiken zweier gesellschaftlicher Sphären - der Sphäre des Marktes für Waren und Dienstleistungen einerseits und der durch individuelle und familiale Solidarität, durch Organisationen der Wohlfahrtspflege sowie durch staatliches Handeln bestimmten sozialen Hilfesysteme andererseits - lässt sich dabei nämlich kaum vermeiden. Ein rein ökonomischer Kundenbegriff lebt von dem idealtypischen Modell (um nicht zu sagen: der Fiktion) autonomer Rechtssubjekte, die sich auf einem anonymen Markt begegnen. Die Umsetzung eines solches Modells in der Realität hat aber etliche Voraussetzungen:

- eine Wahlmöglichkeit zwischen verschiedenen Anbietern,

- die direkte Bezahlung der nachgefragten Dienstleistung,

- die Fähigkeit des Kunden, durch seine Wahl auf die Qualität und den Inhalt des Angebotes Einfluss zu nehmen,

- die Notwendigkeit für den Leistungsanbieter, Abnehmer zu finden, die mit entsprechenden Marketinginstrumenten umworben werden.

Personen, die als „Kunden“ oder „Konsumenten“ der Dienstleistung „vollstationäre Altenhilfe" auftreten, erfüllen anders als Kunden von Hotels, Fitnessstudios oder Beratungsfirmen nur teilweise diese Merkmale. Pflegebedürftige Menschen können in der Regel nur wenig Einfluss nehmen auf die Qualität und den Inhalt des Angebotes, für welches sie sich entscheiden (falls diese Entscheidung nicht überhaupt von Dritten, etwa den Angehörigen, getroffen wird). Oft sind sie ihrer physischen und psychischen Situation wegen gar nicht fähig, die Qualität von Leistungen zu beurteilen. Weiterhin befinden sie sich in einer existentiellen Abhängigkeit von dem Anbieter, wie sie Kunden nur selten erleben. Nur in seltenen Fällen verfügen sie über echte Alternativen. Hinzu kommt die beschränkte Transparenz jener Einzelleistungen, die sich zur Gesamtleistung „vollstationäre Pflege“ addieren. Das Angebot einer Pflegeeinrichtung ist, bevor es in Anspruch genommenen wird, für den Pflegebedürftigen nur wenig konkret und transparent.

Angesichts dieses asymmetrischen Machtverhältnisses zwischen Dienstleistern und ihren „Kunden“ stellt sich die Frage, ob nicht betriebswirtschaftliche Rhetorik hier nur der Legitimation einer sich unter wachsenden Finanznöten verschlechternden Praxis dient: während sich die Versorgung pflegebedürftiger älterer Menschen tendenziell verschlechtert und die Belastungen für das dort engagierte Personal wachsen, werden durch umfangreiche Kontrollprozeduren 
und Berichtspflichten, die einen erheblichen Teil der ohnehin knappen Arbeitsressourcen binden, „Potemkinsche Dörfer“ einer qualitativ hochwertigen Altenpflege aufgebaut. Im Folgenden soll ein bestimmter Aspekt von Qualitätskontrolle und Kundenorientierung in stationären Pflegeeinrichtungen mit Hilfe empirischer Daten untersucht werden: Zunehmend werden Bewohner selber zur Qualität der Pflege und anderer Versorgungsleistungen befragt (vgl. z.B.: http://www.metrik.org/altenheimbefragung/befragung-im-altenheim.htm). Hierbei werden entweder aus der Marktforschung kommende Instrumente zur Messung von Kundenzufriedenheit eingesetzt oder es wird versucht, ähnliche Instrumente für die Altenpflege zu entwickeln. Während manche größeren Träger eigene Fragebögen entwickeln, greifen andere auf vorliegende Messinstrumente zurück (etwa Chou u.a. 2002; Bendel u.a. 2002), die durch standardisierte Fragen Bewohnerzufriedenheit und Pflegequalität messen sollen. Wie brauchbar sind aber solche Verfahren und in welcher Weise lassen sie sich sinnvoll in Strategien der Qualitätssicherung einbeziehen, bei denen reale Probleme in der stationären Altenpflege bearbeitet und gelöst werden müssen?

\section{Kundenbefragungen in stationären Pflegeeinrichtungen - Grundlagen, Ziele und Methoden einer empirischen Studie}

Dieser Frage bin ich anhand einer eigenen empirischen Studie nachgegangen, mit deren Hilfe erkundet werden sollte, wie dauerhaft institutionalisierte ältere Menschen Befragungen erleben (Kelle/Niggemann 2002, 2003). Bevor unsere Methoden und Ergebnisse dargestellt werden, sollen aber zuerst einige Probleme aufgezeigt werden, die eine Befragung dauerhaft institutionalisierter, pflegebedürftiger Menschen mit sich bringt.

\subsection{Die Befragung dauerhaft institutionalisierter älterer Menschen - Diskussionsstand und Probleme}

Kritik an einer empirischen Sozialforschung, die primär an der unmittelbaren Verwertbarkeit ihrer Ergebnisse interessiert ist, ist nichts Neues. Bereits vor etlichen Jahrzehnten monierte Theodor W. Adorno, dass deren gängige Methoden, wie Fragebogen und Interview, die „gesellschaftliche Objektivität, den Inbegriff all der Verhältnisse, Institutionen, Kräfte, innerhalb dessen die Menschen agieren" (Adorno 1972: 84), ignorieren würden. Sozialforschung nehme „das Epiphänomen, das, was die Welt aus uns gemacht hat, fälschlich für die Sache selbst..." (ebd.) und werde somit „Ideologie, notwendiger Schein (...); notwendig, weil ihr Gegenstand, die Gesellschaft, nichts so sehr fürchtet, wie beim Namen gerufen zu werden“ (ebd.: 90). Dennoch, so Adorno, besitze empirische Forschung das Potential, den Schleier der Ideologie, der über den Verhältnissen liegt, zu heben, indem „sie durch Verfeinerung der Methoden 
die Unmittelbarkeit der Daten aufzulösen vermag“ (ebd.: 99). Sozialforschung muss also keinesfalls zur unreflektierten Reproduktion gesellschaftlicher Herrschafts- und Gewaltverhältnisse führen, sondern kann vielmehr nutzbar gemacht werden als Werkzeug zu deren Kritik.

Hierbei muss allerdings als Erstes berücksichtigt werden, dass bei einer wissenschaftlichen Befragung nicht einfach Informationen von einem ansonsten passiven Datenlieferanten abgerufen werden. Vielmehr beteiligen sich Interviewer und Befragter an einem interaktiven Prozess, bei dem sie aufgrund ihrer jeweiligen Motive und Interessen handeln, das Handeln des jeweils anderen einschätzen und ihre Handlungen aufeinander abstimmen müssen. Wie in jeder sozialen Interaktion kann es dabei zu Missverständnissen kommen. Bei dem Versuch, ihre eigenen Ziele durchzusetzen, können Akteure zudem ihre Interaktionspartner über ihre Absichten im Unklaren halten, bestimmte Sachverhalte verschleiern oder verschweigen, Informationen zurückhalten usw. Sowohl die im Alltagsleben verbreiteten unbeabsichtigten Missverständnisse als auch bewusste Täuschungen und Auslassungen können die Aussagekraft von Befragungsergebnissen beeinträchtigen. In unserem Untersuchungskontext ist darüber hinaus zu berücksichtigen, dass sich Interaktionen in Pflegeheimen systematisch von Interaktionen in anderen Befragungskontexten unterscheiden.

Insbesondere Heimbewohner betrachten eine sozialwissenschaftliche Befragung oft als einen willkommenen Anlass zu sozialer Interaktion (Salaske 1997). Solche Befragte wollen dann zwar gerne mit einem Interviewer sprechen, aber oft über andere Themen und in anderer Form, als es standardisierte Fragebogeninstrumentarien mit ihrer strikten Abfolge von Fragen festlegen (vgl. Parker u.a. 1998). Interviewer wiederum empfinden eine standardisierte Befragung älterer Menschen deshalb oft als besonders schwierig und anstrengend, weil diese häufig vom Thema abweichen, direkte Fragen durch Abschweifungen umgehen und Antworten geben, die nicht den vorgeschlagenen Kategorien entsprechen (Rodgers/Herzog 1987). Hinzu kommt, dass physiologische Abbauprozesse im Alter einen Verlust kognitiver Leistungsfähigkeit und sensorischer Fähigkeiten bewirken können, wovon Pflegeheimbewohner natürlich besonders betroffen sind. Befragte mit einer mittleren oder fortgeschrittenen Demenz sind oftmals kaum in der Lage, ein Alltagsgespräch zu führen, geschweige denn, auf die komplexen Fragen eines standardisierten Fragebogens zu antworten.

Schließlich können die Ergebnisse eines sozialwissenschaftlichen Interviews stark verzerrt werden durch die Tendenz, sich dem Interviewer in einer möglichst vorteilhaften Weise zu präsentieren. Bei Skalen zu sozial erwünschtem Antwortverhalten erzielen ältere Menschen im Allgemeinen höhere Werte als jüngere und zeigen auch eine starke Tendenz, negative und extreme Antwortmöglichkeiten zu meiden (vgl. Kühn/Porst 1999). Die Stärke dieser Tendenz ist allerdings abhängig von der Art der Fragen und dem institutionellen Kon- 
text, in dem sie gestellt werden. So meint etwa Laga (1999), dass Heimbewohner bei Befragungen oft sehr angepasst, ängstlich und bescheiden reagieren und führt dies darauf zurück, dass die unnatürliche Interviewsituation die Tendenz zu sozial erwünschtem Antwortverhalten erhöht. Auch wird in der Literatur gelegentlich die Vermutung geäußert, dass gerade dauerhaft institutionalisierte ältere Menschen, die ja von der sie versorgenden Institution stark abhängig sind, bei einer Evaluationsbefragung eher positive als negative Urteile über die Institution äußern werden, um Sanktionen zu vermeiden (so etwa Knäuper u.a. 2002; für den Bereich der ambulanten Pflege kommen Wingenfeld/Schaeffer $2001 \mathrm{zu}$ ähnlichen Überlegungen).

\subsection{Ziele und Methoden des Projektes}

Mit unserem Forschungsprojekt wollten wir einerseits Dimensionen der $\mathrm{Zu}$ friedenheit von Pflegeheimbewohnern und andererseits typische Methodenprobleme bei der Befragung älterer Heimbewohner identifizieren. Hierzu wurden in einem ersten Schritt vierzig qualitative Leitfadeninterviews mit Bewohnern und Bewohnerinnen vollstationärer Pflegeeinrichtungen Norddeutschlands (kirchliche Träger und Träger der freien Wohlfahrtspflege, öffentlichrechtlich und privat) geführt, welche über Kapazitäten von 20 bis 150 Betten verfügten (zum genauen Aufbau der Stichprobe vgl. Kelle/Niggemann 2002). Im zweiten Teil der Untersuchung, bei dem wir ein standardisiertes Instrument zur Messung von Bewohnerzufriedenheit verwendeten, wurde einerseits nach dem Schneeballprinzip eine Stichprobe von Bewohnern von 13 vollstationären Pflegeheimen in ganz Deutschland gezogen $(\mathrm{n}=128)$ und andererseits eine Vollerhebung in einer einzelnen kirchlichen Einrichtung $(\mathrm{n}=116)$ in einer norddeutschen Kleinstadt durchgeführt, bei der jeder Heimbewohner durch einen Interviewer aufgesucht wurde (wobei das Interview dann in vielen Fällen wegen der gesundheitlichen Situation des Befragten oder seiner mangelnden Kommunikationsfähigkeit gar nicht begonnen oder ggf. abgebrochen wurde). Thema der standardisierten Befragung waren die allgemeine Zufriedenheit mit dem Leben in der Pflegeeinrichtung, das Beschäftigungs- und Freizeitangebot, die Pflegeleistungen und die Freundlichkeit und Kompetenz der Mitarbeiter. Im Unterschied zu üblichen standardisierten Interviews, bei denen Befragtenäußerungen, die nicht in das vorgegebene Antwortkategorienraster passen, ignoriert werden, haben wir bei unserer Befragung Kommentare der Befragten, zusätzliche Erzählungen usw. schriftlich protokolliert. Zusätzlich wurden sechzig der Interviews auf Tonträger aufgezeichnet und anschließend teilweise verschriftlicht. 


\section{Kundenbefragungen in stationären Pflegeeinrichtungen - Ergebnisse}

In unserer Studie verfügten wir also erstens über vollständig transkribierte qualitative Leitfadeninterviews, zweitens über quantitative Daten, die mit standardisierten Fragebögen erhoben worden waren, und drittens über verschriftlichte Interaktionsprotokolle der standardisierten Befragung. Die Verknüpfung dieser unterschiedlichen Datenquellen und ihrer Ergebnisse erlaubte es nun, drei zentrale Probleme in den Blick zu nehmen, die bei der Messung von Kundenzufriedenheit in stationären Pflegeeinrichtungen entstehen:

- Die in stationären Einrichtungen oft vom Pflegepersonal unterstützte gezielte Auswahl von Befragten führt zu erheblichen Stichprobenverzerrungen.

- In Befragungen zu ihrer Zufriedenheit zeigen Heimbewohner eine starke Tendenz zu sozial erwünschtem Antwortverhalten.

- Ältere Menschen in institutioneller Betreuung neigen zudem aus Angst vor Sanktionen sowohl zu offenen, als auch zu verdeckten Formen von Verweigerung.

\subsection{Stichprobenverzerrungen bei Befragungen in Pflegeheimen}

Bei Befragungen in Heimen werden Interviewpartner oftmals vom Pflegepersonal ausgewählt. Sowohl Merkmale, die aus Sicht des Personals zu einer guten Befragbarkeit führen (wie das Fehlen sensorischer oder mentaler Einschränkungen), als auch eine unterstellte Befragungsbereitschaft können dann dazu führen, dass bestimmte Bewohner in die Rolle des „Dauerbefragten“ geraten, wie der folgende Ausschnitt aus einem unserer qualitativen Interviews zeigt:

Befragte: Und was soll ich da vorne bei denen (gemeint sind andere Bewohner) sitzen? Nee, ich lese ein bisschen.

Interviewer: Ist das nicht so Ihr Ding?

Befragte: Nee. Die sind doch alle ganz durcheinander. Sonst wären Sie nicht zu mir gekommen. Mit den anderen ... ich hab das schon öfter machen müssen. Auch Praktikantinnen, die sich was aufschreiben müssen. Da musste ich das denn auch so erzählen, ne? Das kenn ich schon, ich hab Lehrlinge ausgebildet, die mussten immer ihre Halbjahresberichte machen. Interview 12.04.01 A04 (453/464)

In jeder Einrichtung ist aber auch ein hoher Anteil von Bewohnern aufgrund von Demenzerkrankungen oder anderer Beeinträchtigungen nicht befragbar. Aus welchen Gründen Bewohner nicht an einer Befragung teilnehmen und wie hoch der Anteil der Nichtbefragbaren tatsächlich ist, lässt sich aber nur dann feststellen, wenn tatsächlich jeder Bewohner kontaktiert wird. Wir hatten großes Glück, eine Einrichtung in kirchlicher Trägerschaft als Kooperationspart- 
ner gewinnen zu können, in der wir eine solche Vollerhebung durchführten. Von den 116 Befragten war ein nennenswerter Teil gar nicht ansprechbar und etliche Personen brachen aus verschiedenen Gründen, zum Teil, weil sie offensichtlich überfordert waren, das Interview ab. Die übrigen Teilnehmer(innen) der Vollerhebung gaben nun häufiger negative Bewertungen über ihre Einrichtung ab als die Teilnehmer(innen) unseres „Schneeballsamples“, bei denen eine Auswahl der Befragten durch das Pflegeheim vorgenommen wurde.

Grafik 1: Vergleich von Ergebnissen aus der Vollerhebung in einer Einrichtung mit Befragungen ausgewählter Heimbewohner

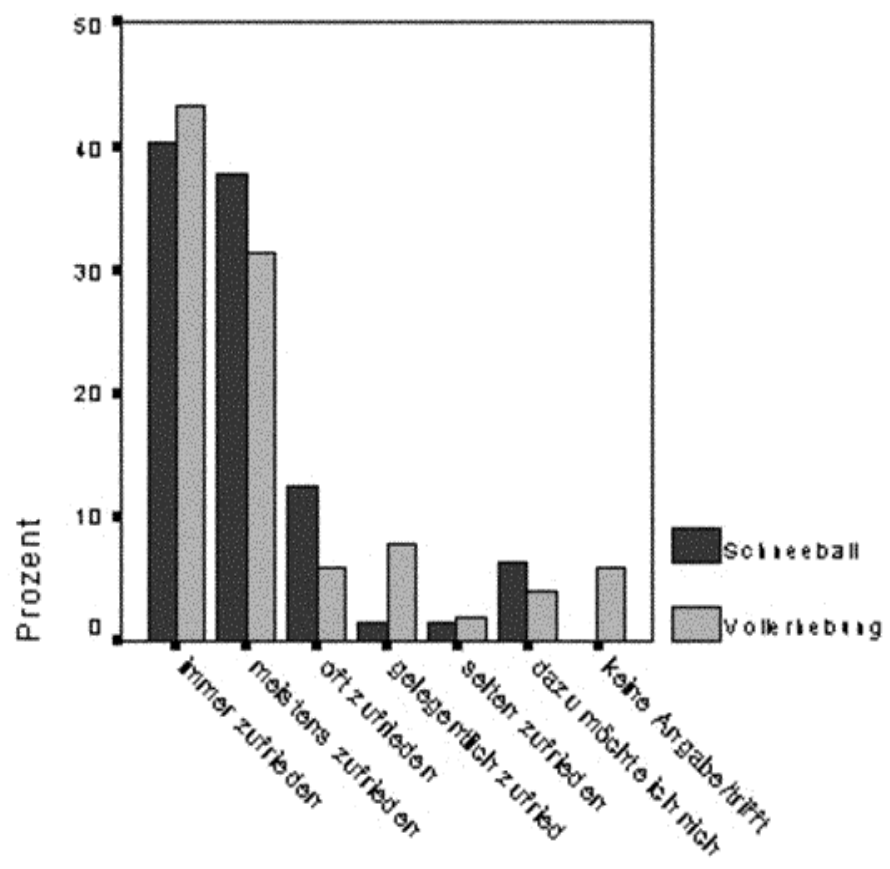

\section{Zufrieden $m$. P flege}

Wie stark die Befragtenauswahl durch die Einrichtung verzerrt sein kann, wurde auch anhand von Feldbeobachtungen deutlich: So wurde beispielsweise einem Heimleiter nach einer ersten Auswertung dreier qualitativer Interviews in seinem Haus mitgeteilt, dass diese Bewohner Einrichtung und Mitarbeiter nahezu uneingeschränkt gelobt hatten. Auf die (eher scherzhaft gemeinte) Frage, ob nur die "freundlichsten und nettesten Bewohner" ausgewählt worden waren, wurde sofort die Möglichkeit zu einem zweiten Besuch angeboten, bei 
dem „kritischere Bewohner“ zum Zuge kommen würden. Der Heimleiter räumte ein, für den ersten Termin „erst einmal die netten und gut gestellten Bewohner“ ausgewählt zu haben. „Man weiß ja schließlich als Einrichtungsleiter, welche Bewohner eher kritisch eingestellt sind, und welche Bewohner das Haus ausschließlich positiv sehen und darstellen." Für den kommenden Termin würde er dann nach Möglichkeit „einige der Herren zur Verfügung stellen, die wären hier in der Einrichtung wesentlich kritischer ...". Auch wenn der anfänglichen Auswahl von „netten“ Bewohnern keinerlei Täuschungsabsicht zugrunde lag, was sich an der Bereitschaft zeigte, auch Gespräche mit „kritischen" Bewohnern zu vermitteln, macht dieses Beispiel deutlich, wie stark Leitung oder Mitarbeiter in dem Bedürfnis handeln, bei einer empirischen Studie, auch wenn diese nicht der konkreten Evaluation einer Einrichtung dient, das Haus und seine Arbeit positiv zu präsentieren.

\subsection{Das Problem des sozial erwünschten Antwortverhaltens}

Die statistische Auswertung der standardisierten Fragebögen vermittelte auf den ersten Blick das Bild großer Zufriedenheit. So wählten etwa drei Viertel bei der Frage „Sind Sie mit der pflegerischen Versorgung durch die Schwestern und Pfleger zufrieden?" die Antwortkategorien „immer“" oder „meistens“. Nur eine äußerst kleine Gruppe bezeichnete sich als nur „gelegentlich“ oder „selten“ zufrieden und niemand antwortete mit „nie zufrieden“. Dies entspricht einer auch in der Literatur beschriebenen Tendenz von Krankenhauspatienten und Heimbewohnern, sich positiv über die Einrichtung zu äußern, in der sie sich aufhalten (vgl. auch Krentz/Olandt 1999).

Die große Mehrheit der Befragten (etwa 55 \% im „Schneeballsample“ und ca. $42 \%$ in der Vollerhebung) stimmten auch der Aussage „völlig“ zu, dass sie mit dem Leben in der Pflegeeinrichtung rundum zufrieden seien. Auch hier ist es nur eine kleine Gruppe, die der Aussage nur „teils-teils“, „wenig“ oder „gar nicht" zustimmt. Eine mögliche Erklärung für die Unterschiede zwischen der Vollerhebung und der Befragung mit ausgewählten Bewohnern wäre natürlich, dass in der Einrichtung der Vollerhebung Bewohner im Durchschnitt unzufriedener sind als anderenorts. Unsere Feldbeobachtungen und der ansonsten sehr positive Ruf dieser Einrichtung legen jedoch den Schluss nahe, dass die Unterschiede eine Folge der Auswahl befragungsfähiger Heimbewohner durch das Personal im Schneeballsample darstellen. Diese Interpretation wird weiterhin gestützt durch die Tatsache, dass der Anteil der Antwortverweigerer an den Befragten in der Vollerhebung mit fast 15\% wesentlich höher lag als bei den ausgewählten Befragten (wo er nur $2 \%$ betrug).

Die in der Literatur gelegentlich geäußerte Vermutung, dass dauerhaft institutionalisierte ältere Menschen dazu tendieren, auf Fragen zu ihrer Zufriedenheit im Sinne eines sozial erwünschten Antwortverhaltens zu reagieren (vgl. Kühn/ 
Porst 1999), ließ sich durch die Verwendung der schriftlichen Interaktionsprotokolle der standardisierten Befragung erhärten. Dabei gab es zahlreiche Hinweise, wie stark sich viele Befragte um ein konformes Interviewverhalten bemühten. Hier wurden nicht nur Andeutungen gemacht, dass Hilfestellungen über die „angemessenen“ und „richtigen“ Antworten erwartet wurden (,...ja was soll ich denn noch sagen?"“, „könnte man halb ja und halb nein sagen?"), mancher Befragte brachte offen den Wunsch zum Ausdruck, sich als guter, kooperationswilliger Interviewter zu erweisen und im Sinne sozialer Erwünschtheit zu antworten: „Wie die das sehen wollen, nich. Wie man das sagen soll."

Entscheidungstheoretischen Ansätzen zufolge (vgl. Esser 1986; Salaske 1997) wägen Befragte den subjektiv erwarteten Nutzen einer Interviewteilnahme mit möglichen Nachteilen ab. Falls solche Kosten-Nutzen-Abwägungen tatsächlich eine wesentliche Rolle bei einer (mehr oder weniger sozial erwünschten) Antwort spielen, so wird dabei auf jeden Fall die Einschätzung des Pflegemarktes und der Situation in anderen Einrichtungen bedeutsam sein. Schwierigkeiten bei der Beschaffung eines Heimplatzes erzeugen oder verstärken ein Abhängigkeitsgefühl der Einrichtung gegenüber, wie das folgende Beispiel zeigt:

Befragte: (...) Und die haben mir auch den Platz hier besorgt. Da war auch eine Verwandte von meiner Schwester hier gewesen, die ist auch hier gestorben, ich weiß aber nicht, wie die heißt. Und überall die Heime voll, die sind ja voll, man kann ja nicht von heute auf morgen sagen, ich will ins Heim. (...) Dann wollte ich vorne nach W., und da war alles belegt, auch. Und jetzt bin ich - habe ich hier den Platz gekriegt, ganz nett, wohl war. Ist eben nicht zu Hause, aber was soll man machen. Das ist nicht zu Hause. (flüstert) Immer schön artig sein.

Interview 20.02.01 B05 (127/136)

Diese Befragte ist weit entfernt von Kundensouveränität oder von Wahlmöglichkeiten auf einem „Pflegemarkt“; sie muss vielmehr zufrieden sein mit dem, was sie (bekommen) hat, da keine Alternativen zur Verfügung stehen. Und sie befürchtet Sanktionen, wenn sie negative Bewertungen über die Einrichtung offen zum Ausdruck bringt. Die geflüsterten Worte sind offensichtlich nur für die Ohren der Interviewerin und nicht für die der Heimleiterin, die sich zur Zeit des Interviews in der Nähe aufhält, bestimmt.

\subsection{Offene und verdeckte Verweigerung}

Bewohner, die vom Pflegepersonal für ein Interview vorgeschlagen wurden, verweigerten nur in seltenen Fällen offen ihre Teilnahme. Dennoch zeigten sich im Laufe der Gespräche Hinweise für verschiedene Formen ,verdeckter Verweigerung“. Einige der Interviewten hätten aus freier Entscheidung wohl 
nicht an einer Befragung teilgenommen, kommen aber zu der Einschätzung, dass ihnen kaum eine andere Wahl bleibt.

$B$ : Gestern wurde ich noch mal darauf hingewiesen, dass ich heute Damenbesuch kriege, und da hab ich gesagt, lass sie bleiben wo sie ist.

I: Ja. Aber warum?

B: (Überlegt). Weil ich doch vor zwei Jahren schon einmal verhört... Mit meinem Arzt verhört worden bin. Unter denselben Bedingungen wie heute.

I: Ja?

$B$ : So ziemlich.

Interview 12.04.01 A01 (622/634)

Dieser während des gesamten Interviews desinteressiert wirkende Bewohner setzte der Situationsdefinition „wissenschaftliche Befragung“, die der Interviewer ihm angeboten hatte, eine eigene Situationsdefinition entgegen, wie sie für Befragungen in „totalen Institutionen“ eher gebräuchlich ist: ein „Verhörter“ hat keine Möglichkeiten, sich für oder gegen seine Teilnahme zu entscheiden, und er muss erwarten, dass das, was er sagt, gegen ihn verwendet wird. Befragungen, deren Funktion und Ziel für Sozialforscher nahezu trivial sind, können bei älteren und dauerhaft institutionalisierten Menschen offensichtlich ganz andere Assoziationen wecken. Doch führt dieser Umstand nur selten zur offenen Verweigerung, wie in einem Fall, in dem eine über 90jährige Bewohnerin auf das Item „Die Pflege ist tadellos und die Schwestern sind immer ausgesprochen freundlich zu mir“, nachdem sie der Aussage zuerst „völlig“ zugestimmt hatte, sagte: „Die Frage ist riskant, darüber spricht man nicht gern. Die Leute werden doch bestraft, die hier die Wahrheit sagen."

Selbst Interessenvertreter der Bewohner erhalten oft keine zuverlässigen Informationen, wenn sie, um Beschwerden nachzugehen, Bewohner direkt aufsuchen, wie eine Interviewpartnerin, die Mitglied des Heimparlaments war, zum Ausdruck brachte:

B: Wir gehen in die Zimmer auch, haben wir auch schon gemacht, ja. Das hat auch nichts gebracht. (...) Das ist, das geht ja auch so schnell, du stehst da null Komma nichts im Zimmer, die sind erst mal baff, bis die sich gesammelt haben, hat er (gemeint ist: ein anderes Mitglied des Heimparlaments) seine Pfeile losgeschossen. 'Haben sie irgendwelche Beschwerden oder Reklamationen oder Sorgen?' Dann sind die so baff und sagen 'nein'. Und dann ist gut und dann geht er wieder raus. (...) Und, wenn wir unten in dem Konferenzzimmer da sind, dann haben wir da Sprechstunde, da kommt kein Mensch (...). Das bringt doch nichts.

Befürchtungen hinsichtlich einer möglichen Weitergabe von Informationen sind durchaus verbreitet, wie der folgende Interviewausschnitt zeigt: 
I: (...) Denken die alten Menschen, dass ich hinterher mit dem Aufzeichnungsgerät irgendwo hin gehe? (...)

$B$ : Ja, die haben kein Vertrauen. Gibt es hier auch einige, die haben kein Vertrauen, überhaupt wo Sie jetzt das Dingen da haben, nech. Was könnte passieren? Vielleicht wird es dem Chef wieder vorgespielt, dann weiß der ganz genau was die gesagt haben, und so denken die. So ist es.

Interview 26.03.01 F01 (775/786)

\subsection{Die Bedeutung von Methoden der Interviewführung}

Ergebnisse einer Messung von Kundenzufriedenheit in Pflegeheimen mit Hilfe standardisierter Fragebögen sind also mit äußerster Vorsicht zu interpretieren. Oft können Probleme des Heimalltags mit anderen Methoden weit eher thematisiert werden: So sprechen viele Befragte im Laufe des standardisierten Interviews quasi nebenbei problematische Aspekte der Pflegesituation an, die erst mit Hilfe ausführlicher Interaktionsprotokolle aufgezeichnet werden können: „Ich kann nicht immer jemanden rufen zum Waschen, da mach ich halt so gut wie's geht ... “ berichtete eine 94-jährige Bewohnerin, die sich bei der Frage nach der Zufriedenheit mit der pflegerischen Vorsorgung für die Antwortkategorie „völlig“ entschieden hatte, und im Kontext einer anderen Frage beklagt sie sich, das Pflegepersonal „habe ... halt immer wenig Zeit ...". Befragte geben zwar häufig positive Antworten, wenn sie mit einem standardisierten Fragebogen global nach ihrer Zufriedenheit gefragt werden, äußern aber gleichzeitig (sozusagen außerhalb des Protokolls) spezifische Kritik an der Einrichtung. Diese Tendenz ist noch stärker in den informell angelegten Interviewsituationen qualitativer Interviews, bei denen Interviewer Erzählanreize geben, die dem bei Heimbewohnern häufig vorhandenen Wunsch nach Kommunikation und Selbstpräsentation entgegen kommen. In solchen Interviews können problematische Aspekte des Heimalltags ad hoc anhand der Schilderungen kurzer Szenen expliziert werden:

$B$ : (...) und der Herr K., auf den muss man immer aufpassen. Das ist ein lieber, netter alter Herr, aber der regt mich auf. Jeden Mittag, aber auch jeden Nachmittag, wenn ich mich hier eingemuddelt hab' in meiner Decke und lieg in meinem Sessel und es ist zwanzig Minuten nach eins, dann bin ich fest eingeschlafen, denn kommt er hier rein und will zur Toilette.

I: Hat der früher gewohnt in Ihrem Zimmer?

$B$ : Und denn schrei' ich ihn an und sag: Raus, raus!, und denn hat er auch seinen Gehwagen, und denn ist das auch schon passiert, dann ist er vor der Tür hingefallen, denn lag er da (...) nicht, er rief immer um Hilfe, und denn kam ich hier rausgestürzt, und mein Mann kam rausgestürzt, und dann haben wir schnell geklingelt, ich weiß Gott sei Dank, wie man den Alarm macht, hab ich gleich Alarm geklingelt, und denn war die Schwester 
grad am Ende, auf dem fünfziger Flur, das ist hier ganz durch und denn dann noch vorbei und denn so rüber ganz hinten ...

Interview 18.01.01 B01 (37/46)

Deutlicher als in einem standardisierten Fragebogen bilden sich in dieser Erzählung die Wirkungen personeller Unterausstattung und eine problematische architektonische Gestaltung der Einrichtung ab, die zu extrem langen Wegen für das Pflegepersonal führt, ohne dass sich die Befragte hier gedrängt sieht, negative Urteile abgeben zu müssen, deren Äußerung Sanktionen von Seiten des Personals auslösen könnten.

\section{Fazit}

Das Eindringen betriebswirtschaftlicher Konzepte des Qualitätsmanagements in die Altenpflege ist notwendigerweise verbunden mit der Suche nach „harten Indikatoren" und quantitativen Maßzahlen, mit deren Hilfe der output des Pflegehandelns ebenso genau gemessen werden kann wie ein finanzieller Ertrag bei der Produktion und dem Vertrieb von marktgängigen Gütern und Dienstleistungen. Pflegebeziehungen und Pflegehandeln spielen sich aber nicht in vollständig transparenten settings ab, Pflegehandeln produziert „Vertrauensgüter“, deren Qualität schwer zu messen und deren Nutzen äußerst schwierig zu quantifizieren ist.

Auf den ersten Blick mag es nahe liegen, in dieser Situation auszuweichen auf die im Marketing beliebten Konzepte von „Kundenzufriedenheit“, die mit Hilfe von Instrumenten der empirischen Sozialforschung einfach operationalisierbar erscheinen. Eine genauere Betrachtung und empirische Untersuchung solcher Verfahren macht jedoch deutlich, dass die mit der Ökonomisierung des Sozialen verbundene Vermischung von sozialpolitischen und karitativen Interventionen mit betriebswirtschaftlicher Rhetorik, wie sie sich in Begriffen von „Qualitätsmanagement“ und „Kundenorientierung“ ausdrückt, zur Verschleierung von Machtasymmetrien zwischen Pflegebedürftigen und Pflegeinstitutionen führt. Anders als auf den idealen oder auch realen Märkten, die den ökonomischen Gleichgewichtstheoretiker interessieren, hat der „Kunde Heimbewohner" nur eingeschränkte Möglichkeiten, seine Bedürfnisse über ein entsprechendes Nachfrageverhalten zum Ausdruck zu bringen. Das hat weit reichende Folgen für die Konstruktion von Messinstrumenten, die die Qualität der Versorgung durch eine Erfassung von Kundenzufriedenheit erfassen sollen. Heimbewohner interpretieren Befragungen zu ihrer Zufriedenheit mit Pflege- und Versorgungsdienstleistungen oft als ein „Ausfragen“ oder sogar als ein regelrechtes „Verhör“ und vermeiden dann jede Form der Kritik aus Angst vor negativen Sanktionen. Aus diesem Grund sind statistische Aussagen zur Kundenzufriedenheit in der stationären Altenpflege in der Regel in Richtung auf ein zu positives Urteil verzerrt. Insbesondere die beliebten, weil kosten- 
und personalsparenden standardisierten Interviews versagen ganz offensichtlich bei der speziellen Population der besonders vulnerablen und abhängigen älteren Menschen in institutioneller Dauerbetreuung.

Die Anwendung von standardisierten Verfahren zur Messung von „Kundenzufriedenheit" steht somit in Gefahr, gesellschaftliche Missstände und Problemlagen bei der Versorgung pflegebedürftiger älterer Menschen zu verdecken, während im Kontext aktueller sozialpolitischer Debatten das falsche Bild einer umfassenden Zufriedenheit und des Einverständnisses der Betreuten erzeugt wird. Beispiele hierfür lassen sich reichlich finden, so etwa die am 11. November 2004 herausgegebene Erklärung des Bundesgesundheitsministeriums, wonach „aufgrund von Fakten, die die Medizinischen Dienste bei Pflegediensten und in Pflegeheimen festgestellt“ wurden, „über 90 Prozent der Pflegebedürftigen, die von ambulanten oder stationären Pflegeeinrichtungen versorgt werden, mit ihrer Pflege zufrieden“" seien (http://www.bmg.bund.de/nn_669444/DE /Presse/Pressemitteilungen/Archiv/Presse-BMGS-1-2004/PM-11-11-2004-6240,param $=$.html). Unter dem Druck ökonomischer Zwänge und bei Verwendung vermeintlich wissenschaftlich begründeter Messverfahren können hier Potemkinsche Dörfer des modernen, marktwirtschaftlich angepassten Sozialstaates entstehen. Kritische Sozialforschung kann allerdings einen Blick hinter deren Fassaden ermöglichen, indem sie die bei der Befragung von Heimbewohnern auftauchenden Probleme benennt und bessere Instrumente zur Entdeckung und Bearbeitung realer sozialer Problemlagen zu entwickeln hilft. Qualitative Interviews etwa können wesentlich zum Aufbau eines Vertrauensverhältnisses zwischen Interviewer und Befragten beitragen, die dann im Laufe des Erzählens eine vorsichtige Haltung aufgeben und negative Bewertungen der Pflegeinstitution offen legen. Vor einer Technisierung auch dieser Verfahren und einer Verdinglichung der Interviewpartner sollte man sich allerdings hüten: „Vertrauen“ lässt sich in der Regel eben nicht einfach durch von den Interviewern einzuübende Verfahren und Tricks methodisch herstellen, so dass auch qualitative Interviews in Einrichtungen der stationären Altenhilfe und -pflege nicht per se gültigere Ergebnisse produzieren. Sie ermöglichen allerdings mit größerer Wahrscheinlichkeit eine Identifikation von Problemfeldern. Informationen, die vielleicht nur von zwei oder drei Personen über bislang unbekannte Probleme im Verhältnis zwischen Pflegern und Gepflegten genannt werden, können oft von weit höherem Nutzen sein als quantitative Maßzahlen, deren Exaktheit nur Schein ist - und die die Mär von der „Kundenorientierung“ in der Altenpflege statistisch verfestigen. 


\section{Literatur}

Adorno, T.W. (1972): Der Positivismusstreit in der deutschen Soziologie. Neuwied: Luchterhand.

Bendel, K.; Matiaske, W.; Schramm, F.; Weller, I. (2002): Patientenzufriedenheit mit ambulanten Diensten, Ergebnisbericht 2002, http://www.werkstatt-opf.de/berichte.html.

Breitscheidel, M. (2005): Abgezockt und totgepflegt. Alltag in deutschen Pflegeheimen. Berlin: Econ.

Chou, S. C.; Boldy, D. P.; Lee, A. H. (2002): Resident Satisfaction and its Components in Residential Aged Care. In: The Gerontologist, 42 (2), S. 188-198.

Esser, H. (1986): Über die Teilnahme an Befragungen. In: ZUMA-Nachrichten 18, S. 38-47.

Fisk, M.; Wigley, V. (2000): Accessing and interviewing the oldest old in care homes. In: Quality in Aging - Policy, Practice and Research 1, S. 27-33.

Fussek, C.; Loerzer, S. (2005): Alt und abgeschoben. Der Pflegenotstand und die Würde des Menschen. Freiburg: Herder.

Jenrich, H.; Schlichting, R. u. a.: Kritiker in der Kritik. In: Altenpflege 05-2006, S. 50-57.

Kelle, U.; Niggemann, C. (2002): „Wo ich doch schon einmal vor zwei Jahren verhört worden bin...". Methodische Probleme bei der Befragung von Heimbewohnern. In: Motel-Klingebiel, A.; Kelle, U. (Hrsg.): Perspektiven der empirischen Alter(n)ssoziologie. Opladen: Leske + Budrich, S. 99-132.

Kelle, U.; Niggemann, C. (2003): Datenerhebung als sozialer Prozess in der Evaluations- und Wirkungsforschung - das Beispiel „Pflegequalität“. In: Hallesche Beitrage zu den Gesundheitsund Pflegewissenschaften, 2 (13) http://www.medizin.uni-halle.de/pflegewissenschaft/journal/ download.htm.

Knäuper, B.; Schwarz, N.; Park, D. (2002): Selbstberichte im Alter. In: Motel-Klingebiel, A.; Kelle, U. (Hrsg.): Perspektiven der empirischen Alter(n)ssoziologie. Opladen: Leske + Budrich, S. 75-98.

Krentz, H.; Olandt, H. (1999): Zufriedenheit mit der Klinik steigt im Alter. In: Gesundheit und Gesellschaft 2 (12), S. 20-21.

Kühn, K.; Porst, R. (1999): Befragung alter und sehr alter Menschen: Besonderheiten, Schwierigkeiten und methodische Konsequenzen. Ein Literaturbericht. In: ZUMA-Arbeitsberichte 99/03. Mannheim: Zentrum für Umfragen, Methoden und Analysen.

Laga, G. (1999): Zur Befragbarkeit „alter“ Menschen. In: Soziale Arbeit 48, S. 302-306.

Parker, S. G.; Peet, S. M.; Jagger, C.; Farhan, M.; Castleden, C. M. (1998): Measuring Health Status in Older Patients. The SF-36 in Practice. In: Age and Aging, 27, S. 13-18.

Rodgers, W.; Herzog, R. (1987): Interviewing older adults. The accuracy of factual information. In: Journal of Gerontology 42, S. 387-394.

Salaske, I. (1997): Die Befragbarkeit von Bewohnern stationärer Alteneinrichtungen unter besonderer Berücksichtigung des Verweigerungsverhaltens. In: Kölner Zeitschrift für Soziologie und Sozialpsychologie 49 (2), 291-305.

Thiele, C.; Feichtinger, L.; Baumann, U.; Mitmansgruber, H.; Somweber, M. (2002): Der Umzug ins Seniorenheim - Erfahrungen von Senioren und Angehörigen. In: Zeitschrift für Gerontologie und Geriatrie 35 (6), S. 556-564.

Wingenfeld, K. (2003): Studien zur Nutzerperspektive in der Pflege (Erweiterte Neuauflage des Papers P02-118). Bielefeld: Veröffentlichungsreihe des Instituts für Pflegewissenschaft.

Wingenfeld, K.; Schaeffer, D. (2001): Nutzerperspektive und Qualitätsentwicklung in der ambulanten Pflege. In: Zeitschrift für Gerontologie und Geriatrie 34, S. 140-146. 